\title{
Improved stability of live attenuated vaccine gdhA derivative Pasteurella multocidaB:2 by freeze drying method for use as animal vaccine
}

\begin{abstract}
The efficacy of attenuated strain of gdhA derivative Pasteurella multocidaB:2 mutant as a live vaccine to control haemorrhagic septicaemia (HS) disease in cattle and buffaloes has been demonstrated. In order to use P. multocida B:2 mutant as a commercial product, it is essential to optimise its formulation for high viability and stability of the live cells. The effectiveness of freeze-drying process using different protective agentformulations for improving cells viability was explored. Sugar and nitrogen compounds were used as protective agents in freeze-drying and the capability of these compounds in maintaining the viability of mutant $\mathrm{P}$. multocida B:2 during subsequent storage was investigated. A complete loss in viability of freeze-dried mutant $\mathrm{P}$. multocida $\mathrm{B}: 2$ was monthly observed until 6-12 months of storage at $-30{ }^{\circ} \mathrm{C}, 4{ }^{\circ} \mathrm{C}$ and $27^{\circ} \mathrm{C}$ when nitrogen compound or no protective agent was added. Trehalose and sucrose showed significantly high survival rate of 93-95\% immediately after freeze-drying and the viability was retained during the subsequent storage at $-30{ }^{\circ} \mathrm{C}$ and $4{ }^{\circ} \mathrm{C}$. A smooth cell surface without any cell-wall damage was observed for the cells formulated with trehalose under scanning electron micrograph. This study presented a freeze-drying process generating a dried live attenuated vaccine formulation with high stability for commercial applications.
\end{abstract}

Keyword: gdhA derivative Pasteurella multocida B:2 mutant; Protective agents; Freeze drying; Cell viability; Storage; Cell morphology 